\title{
Mamíferos fósiles del Pleistoceno tardío (Rancholabreano) de San Mateo Huexoyucán, Tlaxcala, México
}

\author{
Miguel Sánchez Salinas, Eduardo Jiménez Hidalgo, Carlos Castañeda Posadas
}

\begin{abstract}
Miguel Sánchez Salinas
ccpaleo@gmail.com

Garlos Gastañeda Posadas

Benemérita Universidad Autónoma de

Puebla, Facultad de Ciencias Biológicas. Blvd.

Valsequillo y Av. San Claudio, Edificio BIO-1,

Ciudad Universitaria, Col. Jardines de San

Manuel, C.P. 72570 Puebla, México.

Eduardo Jiménez Hidalgo

Laboratorio de Paleobiología, Instituto de Recursos, Universidad del Mar, campus Puerto Escondido, km 2.5 Carretera Puerto Escondido-Sola de Vega, Puerto Escondido, Oaxaca, México. C.P. 71980.
\end{abstract}

BOL. SOC. GEOL. MEX. 2016

VOL. 68 NO. 3

P. $497-514$

\section{RESUMEN}

Se describen los fósiles de mamíferos pleistocénicos procedentes de la zona de San Mateo Huexoyucán, Tlaxcala, México, alojados en la Colección Paleontológica de la Benemérita Universidad Autónoma de Puebla. Se identificaron nueve especies, de las cuales seis son nuevos registros para el área de estudio (Bison antiquus, cf. Bison occidentalis, Camelops hesternus, Equus mexicanus, Eremotherium laurillardi cf. Glyptotherium floridanum). También se reportan formalmente tres especies que fueron mencionadas en trabajos previos (Cuvieronius hyodon, Equus conversidens y Mammuthus sp.). La presencia de Bison permite asignar una Edad Mamífero Rancholabreana (Pleistoceno tardío) a la asociación faunística. En concordancia con esto, los fósiles proceden de una secuencia de fluvisoles con una edad calibrada de 51798 - 47650 años A.P.

Palabras clave: Mamíferos, Rancholabreano, Tlaxcala, México, Pleistoceno.

\section{ABSTRACT}

In this paper, fossils of pleistocene mammals from the area of San Mateo Huexoyucan, Tlaxcala, Mexico, are described. Right now these fossils are housed in the Paleontological Collection of the Benemérita Universidad Autónoma de Puebla (BUAP). Nine species were identified, and six of them are new records for the study area. Bison antiquus, cf. Bison occidentalis, Camelops hesternus, Equus mexicanus, Eremotherium laurillardi cf. Glyptotherium floridanum). Also, three species which were mentioned in previous works, are formally recorded. (Cuvieronius hyodon, Equus conversidens andMammuthus sp.). The presence of Bison, allows to give a Rancholabrean Land Mammal Age (Late Pleistocene) to the faunal assemblage. In line with this, fossils come from a sequence of fluvisols with a calibrated age of 51798 - 47650 B.P.

Keyzords: Mammals, Rancholabrean, Tlaxcala, Mexico, Pleistocene. 


\section{Introducción}

Han pasado cerca de 400 años desde que los restos fósiles de vertebrados pleistocénicos fueron documentados por primera vez en México. Entre los descubrimientos más notables, debido al tamaño de los huesos, estuvieron los mamuts, reconocidos primero como "gigantes" y posteriormente como "elefantes" (Corona et al., 2005).

El Pleistoceno fue un periodo de grandes cambios ambientales que dieron origen a una gran variedad de ecosistemas, los cuales fueron el hábitat de un gran número de especies, muchas de las cuales se encuentran extintas y cuyos restos están ampliamente distribuidos en todo el país (Arroyo-Cabrales et al., 2002). A esta época geológica corresponde el mayor número de localidades paleontológicas descubiertas (Arroyo-Cabrales et al., 2008).

La edad de mamíferos de Norteamérica conocida como Rancholabreano se define como el intervalo de 210000-160000 a 9500 años A.P., la cual abarca una distribución geográfica desde los $55^{\circ}$ de latitud norte hacia el sur de Norteamérica. Biocronológicamente, inicia con la aparición en el registro fósil del género Bison y finaliza con la extinción de la megafaunapleistocénica (Bell et al., 2004).

$\mathrm{El}$ primer registro de fósiles en Tlaxcala fue publicado por Osborn (1921), quien describió a Rhynchotherium tlaxcalae (Castañeda-Posadas et al., 2009). En la segunda mitad del siglo XX, Guenther y Bunde (1973), tras una serie de investigaciones geológico-paleontológicas efectuadas en los estados de Puebla y Tlaxcala, reportaron para la zona de Valsequillo especies fósiles pertenecientes a los órdenes Carnivora, Proboscidea, Perissodactyla, Artiodactyla y Xenarthra; asimismo, establecieron la estratigrafía del lugar y determinaron una edad pleistocénica para los sedimentos portadores (Castañeda-Posadas et al., 2009).

Por otra parte, Bonilla-Toscano (2011) describió ejemplares rescatados en la barranca de San Mateo Huexoyucán, reportando cuatro taxa: Equus, Bison, Mammuthus y Camelidae, así como la presencia de la familia Megatheriidae, al reportar una escápula de un megatérido (Bonilla-Toscano,
2011). Dichos ejemplares se encuentran en la Colección Paleontológica Zoológica de la Escuela de Biología de la Benemérita Universidad Autónoma de Puebla (BUAPALZ). La presencia de Bison en Huexoyucán sugiere una edad Rancholabreana para el área de estudio.

En 2014, Ramírez-Cruz y Montellano-Ballesteros reportaron el hallazgo de la coraza, cráneo y varios restos postcraneales de dos gliptodontes, uno proveniente de la barranca de San Mateo Huexoyucán, y el segundo de Tamaulipas, designando a ambos como Glyptotherium cylindricum; este es el primer reporte de Glyptotherium para Huexoyucán. Dado que hasta ahora sólo se ha publicado formalmente un taxón de la asociación pleistocénica de San Mateo Huexoyucán, Tlaxcala, este trabajo tiene como objetivos, identificar taxonómicamente el conjunto de mamíferos fósiles que se han colectado en el área de estudio y establecer la edad de la asociación faunística.

\section{Marco geológico regional}

Los sedimentos del área de estudio pertenecen al Bloque Tlaxcala, el cual forma parte de la cuenca Puebla-Tlaxcala; éste se elevó debido a una falla normal (Lermo-Samaniego et al., 2006) y está conformado por secuencias lacustres del Terciario que fueron cubiertas por rocas volcánicas de conos monogéneticos del Cuaternario (Vilaclara et al., 2009). La parte superior está coronada por una secuencia de paleosuelos de naturaleza volcánica que cubre desde el Pleistoceno medio hasta el Reciente (Sedov et al., 2009).

Los sedimentos volcánicos consisten en secuencias de tobas andesíticas, andesitas-basaltos y cenizas; por su parte, los sedimentos lacustres están conformados por una secuencias de tobas, aglomerados, grava volcánica de origen fluvial y capas delgadas de pómez y diatomitas con edades de ${ }^{14} \mathrm{C}$ del Pleistoceno tardío al Holoceno que varía de 46000 a 1420 años A.P. (De la Treja et al., 2014).

El pedocomplejo de la Barranca de San Mateo Huexoyucán se ha clasificado como un fluvisol hís- 


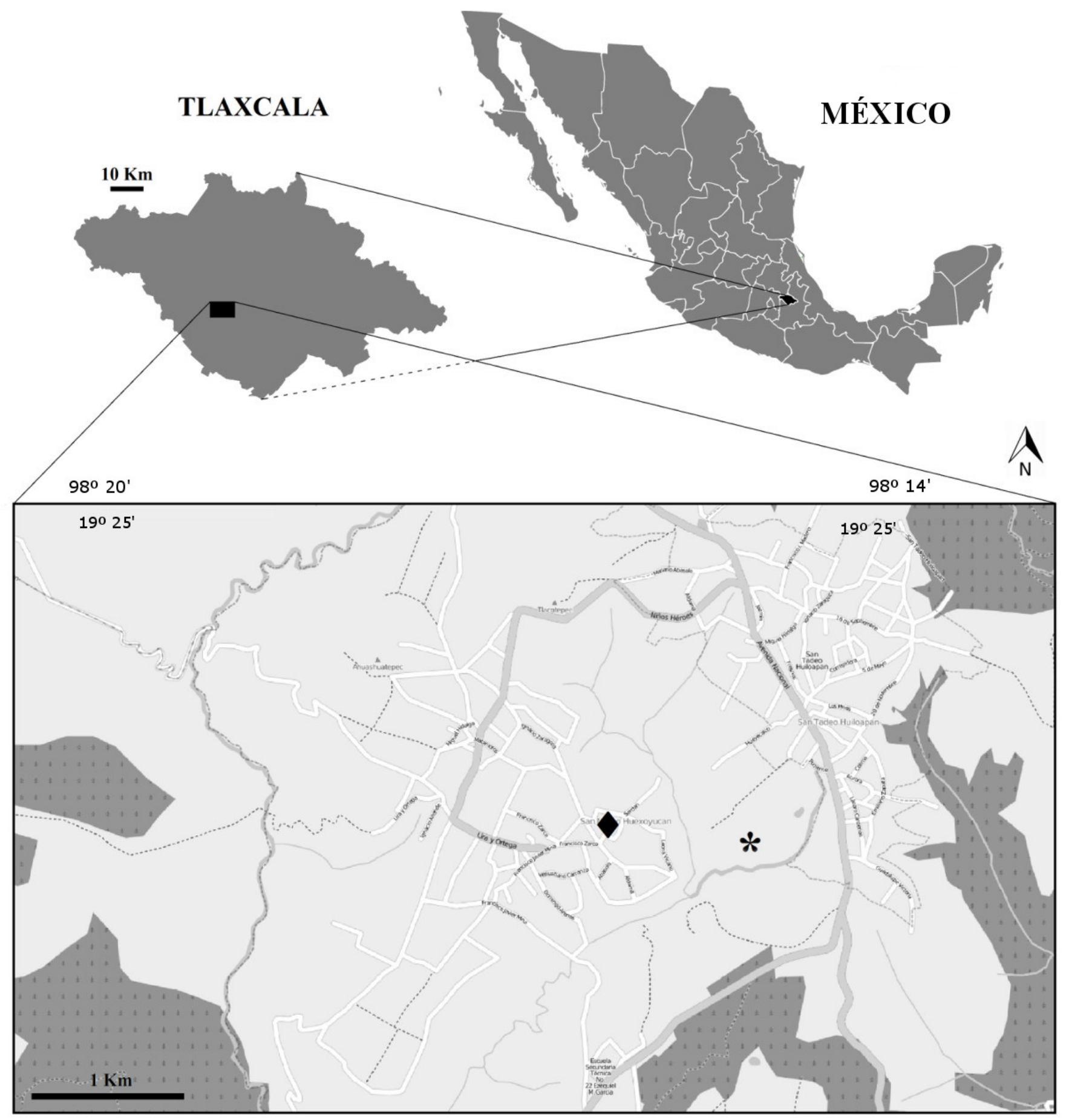

SAN MATEO HUEXOYUCÁN $\diamond$ LOCALIDAD FOSILÍFERA *

Figura 1 Mapa del área de estudio (modificado de Alonso-Aguilar et al., 2014). 
tico (Figura 2) con una datación de radiocarbono estimada en $46320 \pm 870$ años A.P. y una edad calibrada de 51798 - 47650 años A.P. (Tovar et al., 2013). Los paleosuelos tienen una estructura en bloques subangulares; los rasgos reductomórficos predominan, con una alta acumulación de humus y arcilla tanto en la matriz como en la superficie de los agregados, formados por procesos de iluviación, muestran ligeros rasgos de pedogénesis, su textura es limosa con una densidad aparente baja debido al origen volcánico del material (ceniza) (Solís-Castillo et al., 2012). Los niveles fosilíferos forman parte de esta secuencia de fluvisoles, por lo que la edad de la mayoría de los ejemplares aquí descritos se encuentra entre las edades antes mencionadas, con excepción de los osteodermos,

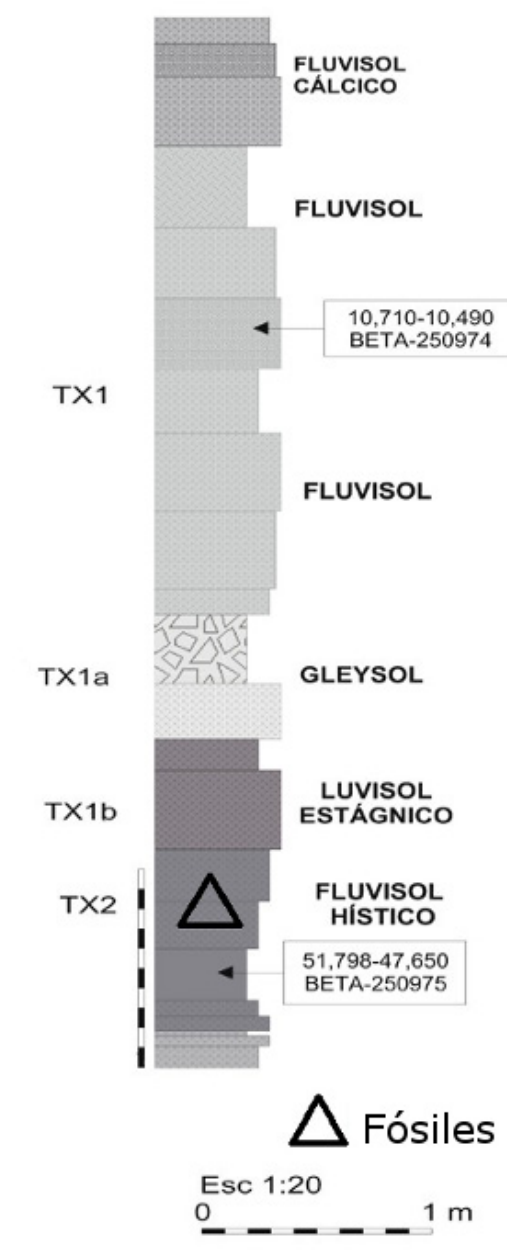

Figura 2 Columna estratigráfica del área de estudio (modificado de Solís-Castillo et al., 2012). los cuales fueron encontrados en el suelo de la barranca.

\section{Material y método}

La exploración y la colecta de los fósiles se realizaron a lo largo de los estratos de las laderas y suelos de la Barranca de San Mateo Huexoyucán. Posteriormente a cada fósil colectado se le asignó un número de inventario BUAPALZ y la localidad de procedencia.

La identificación de la morfología ósea de los ejemplares fósiles se realizó con ayuda del "Atlas de anatomía de los animales domésticos" (Sisson y Grossman, 1978). Para la clasificación de los órdenes de los vertebrados fósiles se consultaron los registros de la megafauna de La Edad de Hielo de la Comisión Nacional para el Conocimiento y Uso de la Biodiversidad (CONABIO, 2012), el Catálogo de Vertebrados Fósiles del Estado de Hidalgo (Castillo-Cerón et al., 1996) y la bibliografía especializada de mamíferos tanto actuales como fósiles (entre los que destacan los trabajos realizados por Gillette y Ray, 1981; De Iuliis, 1996; Martinelli et al., 2012 y Ramírez-Cruz y Montellanos-Ballesteros, 2014). Diversos ejemplares fueron comparados con aquellos de la sección de vertebrados de la Colección Científica del Laboratorio de Paleobiología, campus Puerto Escondido, Universidad del Mar (UMPE).

\section{Resultados}

Se estudiaron un total de 68 ejemplares fósiles procedentes de la Localidad 6 denominada "Barranca de San Mateo Huexoyucán”, Tlaxcala. La mayoría de los ejemplares son fragmentos de huesos largos (fémur, húmero, radio, fíbula, tibia, falanges), escápulas, vértebras, huesos carpianos, tarsianos, osteodermos y molares. Se identificaron cinco órdenes (Artiodactyla, Cingulata, Perissodactyla, Proboscidea, Xenarthra), siete familias (Bovidae, Camelidae, Equidae, Glyptodontidae, 
Gomphotheriidae, Megalonychidae, Elephantidae) y siete géneros (Bison, Camelops, Cuvieronius, Equus, Eremotherium, Glyptotherium, Mammuthus), los cuales se describen a continuación.

\subsection{PALEONTOLOGÍA SISTEMÁTICA}

\author{
Orden Perissodactyla Owen, 1848 \\ Familia Equidae Gray, 1821 \\ Equus Linnaeus, 1758
}

Equus conversidens Owen, 1869

Material examinado. BUAPALZ-1080, M1 derecho; BUAPALZ-1081, M3 derecho, BUAPALZ1082, M1 izquierdo, BUAPALZ-1083, M2.

Descripción. Los ejemplares BUAPALZ-1080 y BUAPALZ-1082, fueron identificados como molares superiores derechos; ambos ejemplares no conservan el cemento, pero sí el esmalte. El ejemplar BUAPALZ-1080, es el más conservado, presenta la prefoseta y la postfoseta; en la parte anterior del diente se conserva el pliprotolofo (Figura 3 A, véase apéndice de abreviaturas anatómicas). El ejemplar BUAPALZ-1081 está fragmentado pero presenta la postfoseta y el plihipostilo (Figura $3 \mathrm{~B}$ ). A pesar del estado del ejemplar BUAPALZ 1082, se conserva la prefoseta y la postfoseta, mientras que el protocono está fragmentado (Figura $3 \mathrm{C}$ ). El ejemplar BUAPALZ-1083 es un molariforme superior derecho sin cemento, conserva la prefoseta, la postfoseta y el protocono, mientras que la corona dental no presenta desgaste, lo que sugiere que perteneció a un ejemplar juvenil (Figura 3 D). Discusión. Los ejemplares estudiados fueron comparados con el ejemplar UMPE -077 cráneo de Equus conversidens con la dentición completa. En los ejemplares de Tlaxcala las prefosetas y postfosetas son complejas, presentan similitud en tamaño y forma con los ejemplares oaxaqueños, por lo que se les asignó a Equus conversidens.

Equus mexicanus Hibbard, 1955
Material examinado. BUAPALZ-1045, porción de premaxilar.

Descripción. BUAPALZ-1045 es un premaxilar de tamaño grande, ancho, sin dientes incisivos, con un diente canino izquierdo preservado, la parte anterior del premaxilar es amplia y ligeramente curva, con un ancho de $69.16 \mathrm{~mm}$ a la altura de los alvéolos de los terceros incisivos (Figura 3 E). Los alvéolos de los incisivos primero y segundo son ovoidales y profundos, el ancho entre los caninos es de $68.84 \mathrm{~mm}$; en el hueso intermaxilar se encuentra un foramen que continúa hasta la parte superior del premaxilar, presenta la apófisis palatina (Figura $3 \mathrm{~F}$ ).

Discusión. Al comparar el premaxilar BUAPALZ-1045 con el ejemplar UMPE-077 (cráneo de Equus conversidens), se observó que era más grande y más ancho, mientras que al compararlo con el cráneo UMPE-521 (Equus mexicanus) ambos mostraron similitud morfológica y en tamaño, por lo que el ejemplar de Tlaxcala se identifica como $E$. mexicanus.

Adicionalmente, la parte anterior del premaxilar es amplia, plana en la superficie ventral en comparación con las formas recientes, y no se curva bruscamente hacia abajo como en el caballo doméstico y la cebra. La superficie dorsal de la parte anterior del premaxilar no se dobla bruscamente hacia abajo, sino que continúa con casi la misma pendiente desde la muesca nasal hasta el alveolo del segundo incisivo, como se observa en el cráneo de E. mexicanus (Hibbard, 1955).

El ejemplar estudiado presenta un diente canino fragmentado y pequeño, lo que permite la identificación del sexo del ejemplar como una hembra (Figura $3 \mathrm{G}$ ).

\section{Equus sp.}

Material examinado. BUAPALZ-1044, fragmento de escápula.

Descripción. Escápula fragmentada, con el acromion roto y la parte del cuello de la escápula fragmentada, presenta la base de la espina esca- 
A
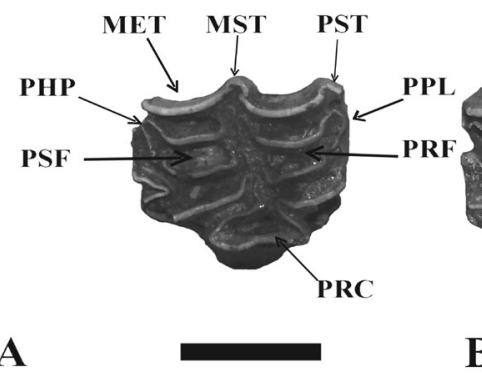

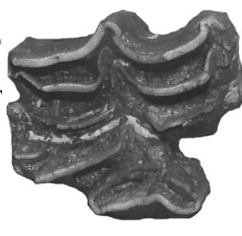

B

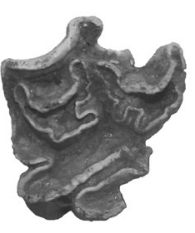

C

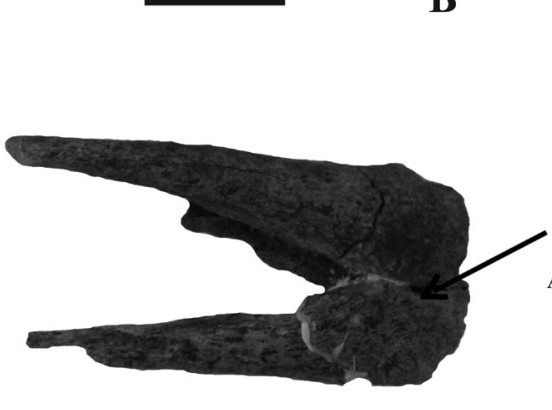

$\mathbf{E}$

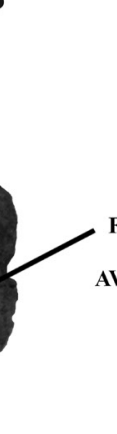

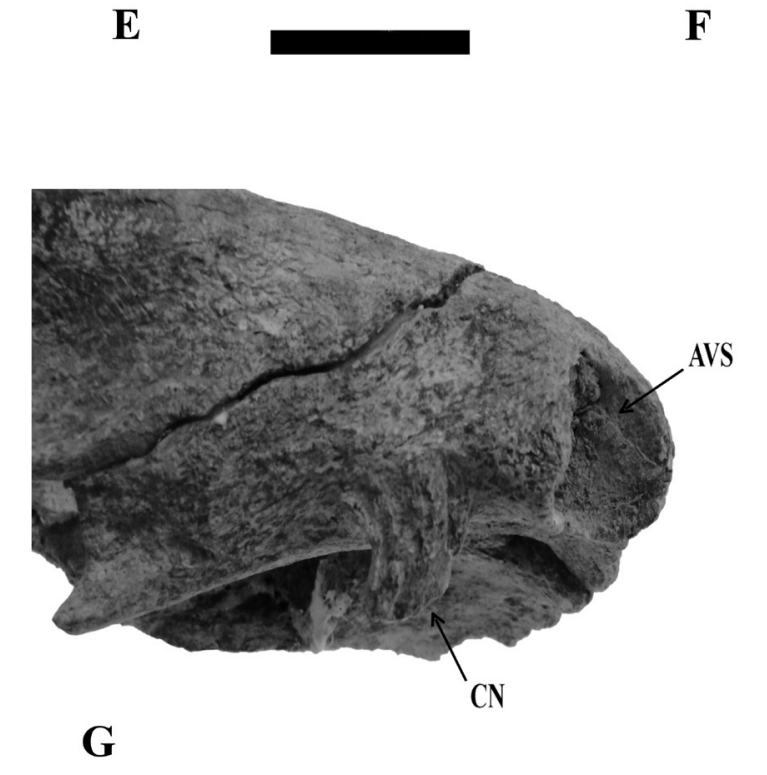

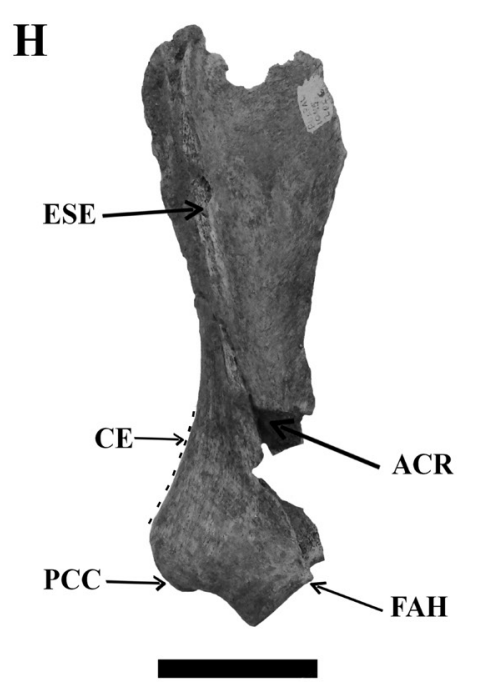

Figura 3 Équidos de la Barranca de San Mateo Huexoyucán, Tlaxcala, México. A, BUAPALZ-1080; B, BUAPALZ-1081; C, BUAPALZ-1082; D, BUAPALZ-1083, molariformes derechos superiores de Equus coversidens; BUAPALZ-1045 premaxilar de Equus mexicanus; E, vista ventral; F, vista dorsal; G, detalle del diente incisivo superior; H, BUAPALZ-1044, escápula fragmentada de Equus sp. En A - D la escala representa $2 \mathrm{~cm}$; en $E-H$ representa $5 \mathrm{~cm}$.

pular, el tubérculo escapular conservado, el proceso coracoideo alargado, con la faceta articular del húmero fragmentada y con forma de corazón (Figura $3 \mathrm{H}$ ).

Discusión. En Equus el tubérculo de la escápula es amplio, como la articulación del húmero, además, el borde lateral de la articulación es plano y redondo (Schmid, 1972), tal como se presenta en el ejemplar descrito.

A pesar del estado fragmentado que presenta el ejemplar BUAPALZ-1044, por el tamaño de la tuberosidad escapular y la articulación del húmero es factible que perteneciera a un ejemplar juvenil de équido. 


\section{Orden Artiodactyla Owen, 1848 \\ Familia Bovidae Gray, 1821}

\section{Bison Smith, 1827 \\ cf. Bison occidentalis}

Material referido. BUAPALZ-1009 y BUAPALAZ-1011, acetábulos fragmentados.

Descripción. El Ejemplar BUAPALZ-1009 es un fragmento de acetábulo amplio y fragmentado. El labio del acetábulo es grande y presenta la muesca acetabular en forma de herradura, profunda y curva. También están preservados la mitad del foramen del acetábulo, con fragmentos del ilion e isquion (Figura 4 A). El ejemplar BUAPALZ-1011, un fragmento de ilion más pequeño y fragmentado, presenta la muesca acetabular, el fragmento del labio es más delgado y no tan pronunciado (Figura 4 B).

Discusión. En los ejemplares BUAPLAZ-1009 y BUAPALZ-1011 se midió LA (véase apéndice abreviaturas de medidas) y fueron comparados con los ejemplares de Bison antiquus UMPE 0464, UMPE 0444 y el ejemplar de Bison occidentalis MMMNV-1914 (Tabla 1). Se encontró que los ejemplares muestran similitud en medidas al ejemplar de Bison occidentalis MMMNV-1914. Ambos ejemplares son más pequeños que los ejemplares de la fauna local VikoVijin reportados por Jiménez-Hidalgo et al. (2013), ya que las pelvis en Bison occidentalis son más cortas y pequeñas que las de Bison antiquus (McDonald, 1981). Por lo anterior, los ejemplares estudiados se asignan tentativamen-

Tabla 1. Medidas del largo del labio acetabular (LA) de los ejemplares de Bison antiquus (UMPE 464 y UMPE 0445) y $B$. occidentalis (MMMNV-1914). Medidas en milímetros ( $\mathrm{mm}$ ).

\begin{tabular}{|c|c|}
\hline Ejemplar & LA \\
\hline BUAPALZ -1009 & 89.46 \\
\hline MMMN V-1914 & 89.6 \\
\hline BUAPALZ -1011 & $50.14^{*}$ \\
\hline UMPE 0464 & 94.69 \\
\hline UMPE 0445 & 100.3 \\
\hline
\end{tabular}

* estimada te a Bison occidentalis, ya que no se tienen ejemplares más diagnósticos, como los núcleos de los cuernos, para su identificación precisa a nivel de especie. Bison occidentalis aparece en el registro fósil a finales del Pleistoceno, por lo que posiblemente los ejemplares BUAPALZ-1009 y BUAPALZ-1011 fueron recolectados de la parte superior de la secuencia estratigráfica del área de estudio.

\section{Bison antiquus Leidy, 1852}

Material referido. BUAPALZ-1046, calcáneo fragmentado.

Descripción. Cuerpo calcáneo esbelto y no tan ancho. A diferencia del calcáneo del caballo, la tuberosidad calcánea termina con una cabeza protuberante; el sustentáculo está fragmentado y presenta el proceso anterior también fragmentado; en la parte inferior presenta una faceta curva y articular para el astrágalo (Figura $4 \mathrm{C}$ ).

Discusión. El ejemplar BUAPALZ-1046 tiene una protuberancia y el proceso anterior termina de forma triangular como en el género Bison (Oslen, 1960; Schmid, 1972). El ejemplar estudiado fue medido y comparado con los calcáneos de Bison antiquus reportados por Raymond y Prothero (2011) y presentó similitud en medidas, específicamente, del largo desde la punta de la tuberosidad del calcáneo hasta la faceta para el astrágalo, con los ejemplares de calcáneo de Bison antiquus de Rancho La Brea reportados por dichos autores (Tabla 2).

Tabla 2. Medida del Largo de tubérculo del calcáneo (LTC) del ejemplar BUAPALZ-1046 y los ejemplares de Bison antiquus de Rancho La Brea reportados por Raymond y Prothero (2011). Medidas en milímetros ( $\mathrm{mm}$ ).

\begin{tabular}{|c|c|}
\hline Ejemplar & LTC \\
\hline BUAPALZ -1046 & 125.2 \\
\hline RLB* & 126.3 \\
\hline RLB* & 125.9 \\
\hline RLB* & 125.7 \\
\hline RLB* & 123.5 \\
\hline
\end{tabular}

* Raymond y Prothero no mencionan los números de catálogo, sólo indican que los ejemplares pertenecen a la colección Rancho La Brea en California. 


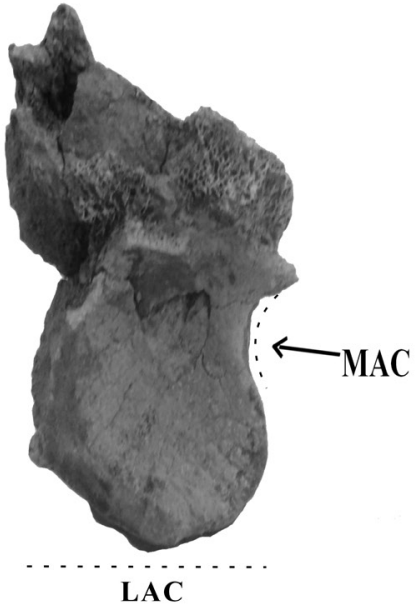

B

LAC

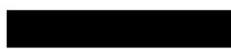

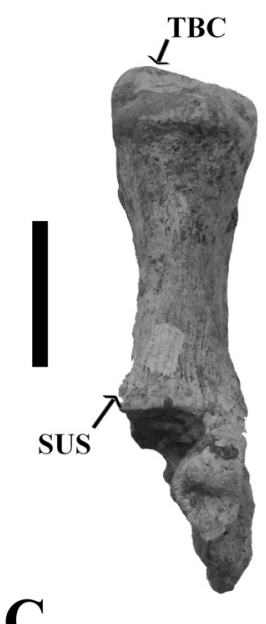

$\mathbf{C}$

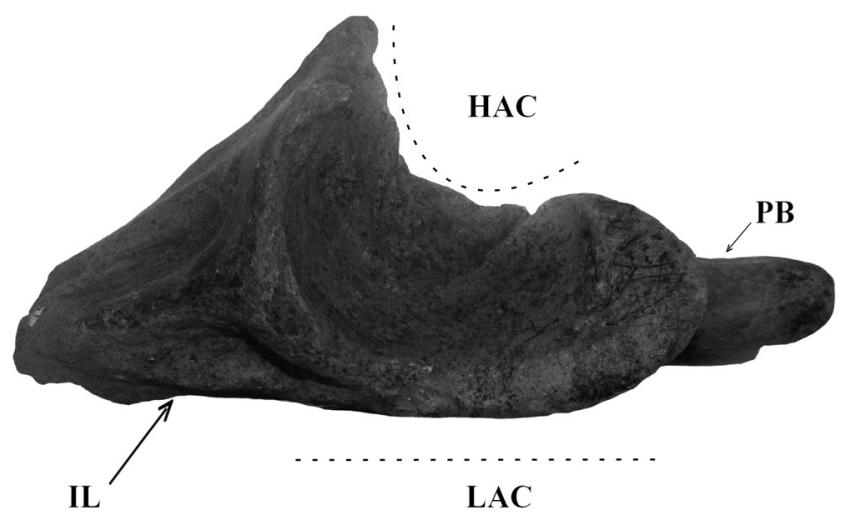

Figura 4 Bisontes Pleistocénicos de la Barranca de San Mateo Huexoyucán, Tlaxcala, México. A, BUAPALZ-1011, fragmento de acetábulo de cf. Bison occidentalis; B, BUAPALZ-1009, fragmento de acetábulo de cf. Bison occidentalis; C, BUAPALZ-1046, calcáneo de Bison antiquus. Escala $=5 \mathrm{~cm}$.

\section{Familia Camelidae Gray, 1821 \\ Camelops Leidy, 1854}

Camelops hesternus Leidy, 1873

Material referido. BUAPALZ-1036, metatarso casi completo.

Descripción. Hueso metatarsiano relativamente robusto, carece de la porción distal, presenta un canal posterior bien definido (Figura $5 \mathrm{Al}$ ), la diáfisis proximal está fragmentada y presenta la face- ta para el hueso cuneiforme alargada y reniforme, separada por una fosa oval poco profunda. La faceta para el entocuneiforme está fragmentada, presenta un pequeño canal que la divide de la faceta del ectomesocuneifome, la cual también está fragmentada (Figura 5 A2).

Discusión. El ejemplar fue comparado con metapodiales de Hemiauchenia y Camelops. Es más grande y más robusto que los ejemplares de Hemiaucheniama crocephala UF 66423 y UTEP 25-202, pero similar a los metatarsos de Camelops (Tabla 3). En una revisión taxonómica reciente sobre Camelops, 
Tabla 3. Medidas del ejemplar BUAPALZ-1036 y de los metatarsales de Camelops y Hemiauchenia. Medidas en milímetros $(\mathrm{mm})$.

\begin{tabular}{|c|c|c|c|c|}
\hline Ejemplar & Largo & $\mathbf{A E}$ & $\begin{array}{c}\mathbf{A T} \mathbf{~ a} \\
\mathbf{1 1 . 5} \mathbf{~ c m}\end{array}$ & $\begin{array}{c}\mathbf{A T} \mathbf{~ a} \\
\mathbf{2 2 . 5} \mathbf{~ c m}\end{array}$ \\
\hline $\begin{array}{c}\text { UF 66423 } \\
\text { Hemiauchenia macrocephala }\end{array}$ & 326.4 & 43.56 & 29.16 & 34.09 \\
\hline $\begin{array}{c}\text { BUAPALZ-1036 } \\
\text { UTEP 25-202 }\end{array}$ & $265 *$ & $66.41 *$ & 41.38 & 40.8 \\
\hline $\begin{array}{c}\text { H. macrocephala } \\
\text { Camelops } *\end{array}$ & 393.8 & 43.52 & 29.22 & 28.1 \\
\hline $\begin{array}{c}\text { (Breyer, 1974) } \\
\text { Camelops hesternus }+ \\
\text { (antes C. traviswhitei) }\end{array}$ & 364 & 67 & -- & -- \\
\hline $\begin{array}{c}\text { Camelops hesternus } \\
\text { (Yukon) }\end{array}$ & 310.9 & 80 & 56 & 50.7 \\
\hline $\begin{array}{c}\text { C. hesternus } * * * \\
\text { (Webb, 1965) }\end{array}$ & $357-388$ & $74-89$ & -- & $40-49$ \\
\hline
\end{tabular}

*Presenta parte de la porción lateral de la epífisis proximal perdida. *:Se presentan los valores promedio. *:*Se presentan el intervalo observado. +De acuerdo con Baskin y Thomas (2016).

A1
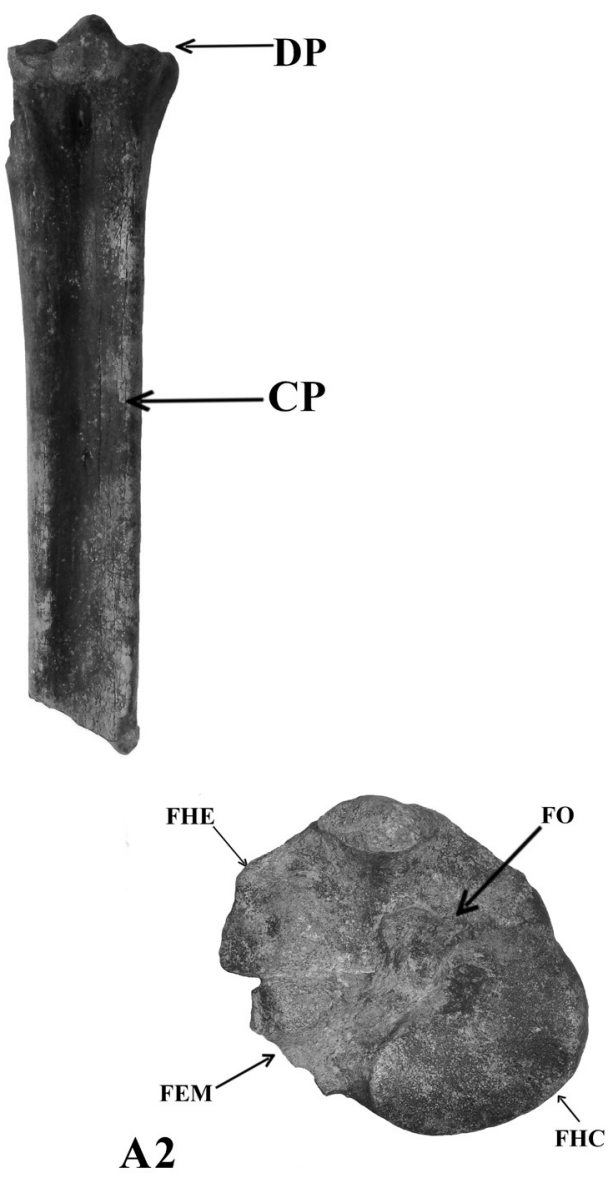

Figura 5 BUAPALZ-1036, hueso metatarsiano de Camelops hesternus. A, Vista craneal; B, cara metatarsiana. Escala $=10$ cm.
Baskin y Thomas (2016) consideran que Camelops hesternus es la única especie de camélido durante el Rancholabreano, por lo que el material aquí descrito se asigna a esta especie.

Orden Proboscidea Illiger, 1811

Familia Elephantidae Gray, 1821

Mammuthus Brookes, 1828

\section{Mammuthus sp.}

Material referido. BUAPALZ-1031, cintura pélvica fragmentada; BUAPALZ-1026, calcáneo. Descripción. Fragmento de cintura pélvica de $60 \mathrm{~cm}$ de largo, con fragmentos del ilion e isquion. El acetábulo es grande y profundo, de $24 \mathrm{~cm}$ de radio, con la hendidura acetabular bien marcada; el foramen obturador es pequeño y ovalado (Figura $6 \mathrm{~A})$.

El calcáneo tiene $30 \mathrm{~cm}$ de largo y $23 \mathrm{~cm}$ de alto, presenta las facetas para el astrágalo y el cuboides preservadas y la tuberosidad del calcáneo completa, la faceta lunar para el astrágalo está fragmentada y no conserva el sustentáculo, la tuberosidad calcánea es cuadrangular (Figura 6 B).

Discusión. Debido a las dimensiones que presenta el ejemplar BUAPALZ-1031 se considera que pertenece a un proboscídeo. El ejemplar fue comparado con los ejemplares DP 5797, DP 5798 y DP 5799, pelvis de Cuvieronius reportados por Tapia-Ramírez et al. (2013) y el ejemplar KP 28689 pelvis de Mammuthus reportado por Baygusheva y colaboradores (2012, Tabla 4).

$\mathrm{Al}$ ser comparados, el diámetro del acetábulo del ejemplar BUAPALZ-1031, es mayor que los ejemplares de Cuvieronius, pero presenta más similitud con el ejemplar de Mammuthus, por lo que se asignó a este género. Adicionalmente, el ilion es amplio en todos los géneros de proboscídeos, el isquion y el pubis son cortos en Mammuthus, lo que da lugar a un foramen obturador pequeño (Tapia-Ramírez et al., 2013) tal como se observa en la pelvis fragmentada BUAPALZ-1031.

En Mammuthus la tuberosidad calcánea es cuadrangular y carece de cuello (Tapia-Ramírez et al., 
Tabla 4. Medidas del diámetro anteroposterior del acetábulo (DA) de la pelvis del ejemplar BUAPALZ-1031 y de los ejemplares reportados por Baygusheva et al., (2012) y Tapia-Ramírez et al., (2013). Medidas en milímetros (mm).

\begin{tabular}{|c|c|c|c|c|}
\hline Ejemplar & DA & Familia & Género & Referencia \\
\hline BUAPALZ-1031 & 204 & Elephantidae & Mammuthus & Este estudio \\
\hline DP 5797 & 170 & Gomphotheriidae & Cuvieronius & Tapia-Ramírez et al ., 2013 \\
\hline DP 5798 & 140 & Gomphotheriidae & Cuvieronius & Tapia-Ramírez et al., 2013 \\
\hline DP 5799 & 75 & Gomphotheriidae & Cuvieronius & Tapia-Ramírez et al., 2013 \\
\hline KP 28689 & 207 & Elephantidae & Mammuthus & Baygusheva et al ., 2012 \\
\hline
\end{tabular}

2013), característica que se observa en el ejemplar BUAPALZ-1026, el cual fue medido y comparado también con el ejemplar de Mammuthus reportado por Baygusheva y colaboradores (2012, Tabla 5). Se observó similitud en medidas y al ser comparado con el Smithsonian 3DX Woolly mammothModel se confirmó la similitud morfológica, pese a la ausencia del sustentáculo del ejemplar estudiado (Metallo et al., 2010).

\section{Familia Gomphotheriidae Cabrera, 1929 Cuvieronius Osborn, 1923}

Cuvieronius hyodon Fischer, 1814

Material referido. BUAPALZ-1062, calcáneo. Descripción. Hueso calcáneo fragmentado, con un cuello bien definido y una tuberosidad calcánea alargada y fragmentada, también presenta el sustentáculo fragmentado (Figura $6 \mathrm{C}$ ).

Discusión. De acuerdo con algunos autores, $\mathrm{Cu}$ vieronius es más pequeño que Mammuthus y Mammut (Lucas y Alvarado, 2010; Tapia-Ramírez et al., 2013). El calcáneo de Cuvieronius presenta la tuberosidad calcánea alargada y sin protuberancias, el sustentáculo es corto y prominente (Tapia-Ramírez et al., 2013). En el ejemplar BUAPALZ-1062 se puede apreciar la tuberosidad calcánea baja y no tan pronunciada como en Mammuthus. Al ser comparado, se observó que el ejemplar BUAPALZ-1062 era más pequeño que en Mammuthus y se encontró similitud en tamaño (Tabla 6) y forma con los ejemplares de Cuvieronius reportados por Tapia-Ramírez y colaboradores (2013), por lo que se identificó como Cuvieronius hyodon.
Orden Xenarthra Cope, 1889

Familia Megatheriidae Gray, 1821

Eremotherium Spillmann, 1948

Eremotherium laurillardi Lund, 1842

Material referido. BUAPALZ-998, metacarpo. Descripción. Metacarpo robusto y alargado de $255 \mathrm{~cm}$ de largo; en la porción proximal presenta la faceta para el unciforme y el metacarpo $\mathrm{V}$, en la porción distal presenta una faceta para la falange proximal redonda (Figura 7).

Discusión. Eremotherium se distingue de Megatherium por la forma del cráneo, los molares y las manos. Las manos de Eremotherium contienen los dígitos III al V, de los cuales los dígitos III y IV son largos, más robustos y bien formados (Cartelle y De Iullis, 1995). El ejemplar BUAPALZ-998 es robusto y al ser comparado con los rangos observados en las dos especies de Eremotherium en América del Norte, se encuentra en el rango observado para Eremotherium laurillardi (226 - $316 \mathrm{~mm})$.

La distribución geográfica de estos perezosos terrestres gigantes va desde el sur de Estados Unidos hasta Brasil, mientras que, Eremotherium eomigrans sólo se ha encontrado y reportado en Florida, Estados Unidos (Cartelle y De Iullis, 1995).

Orden Cingulata Illiger, 1811

Familia Glyptodontidae Gray, 1869

Glyptotherium Osborn, 1903 cf. Glyptotherium floridanum 

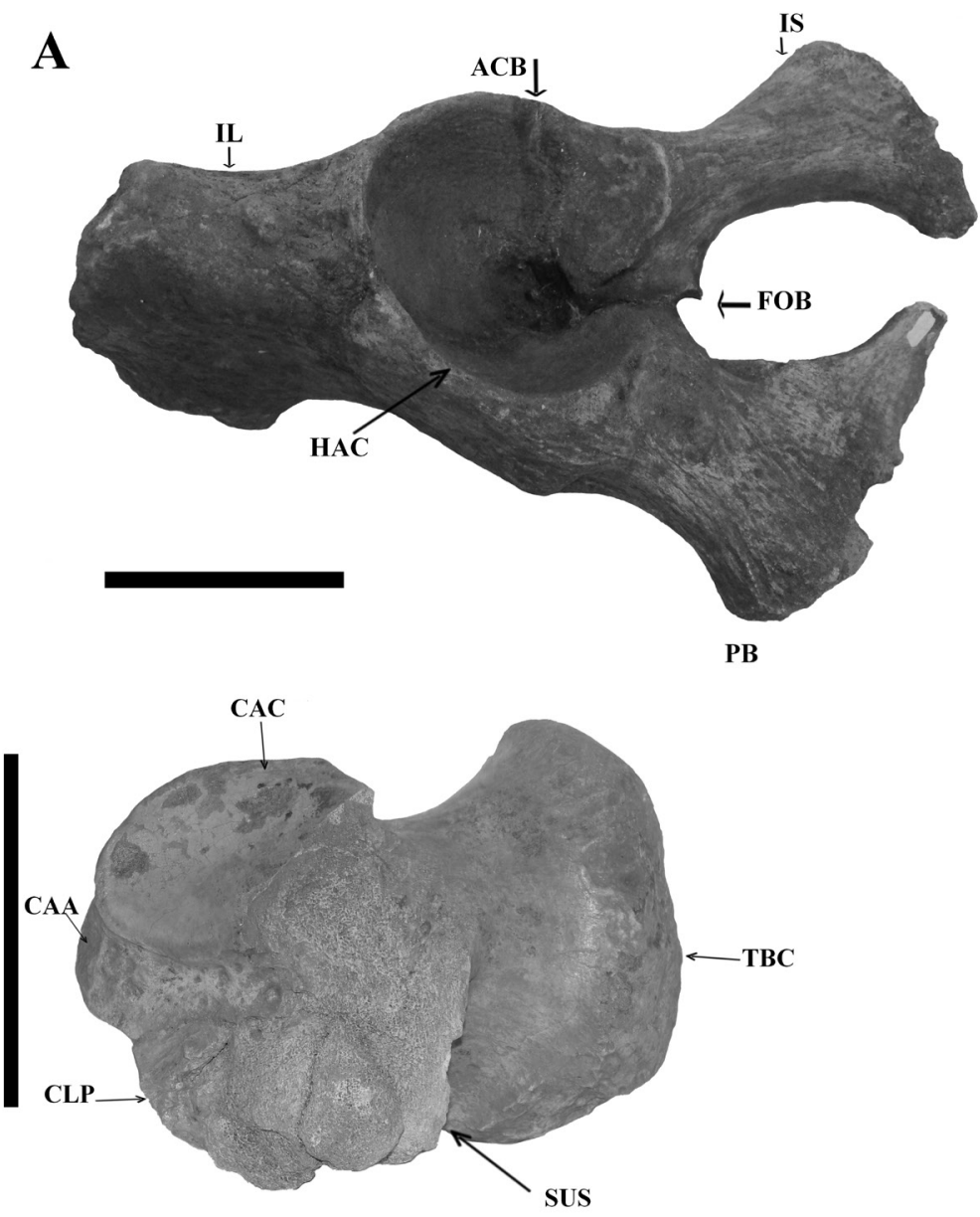

B

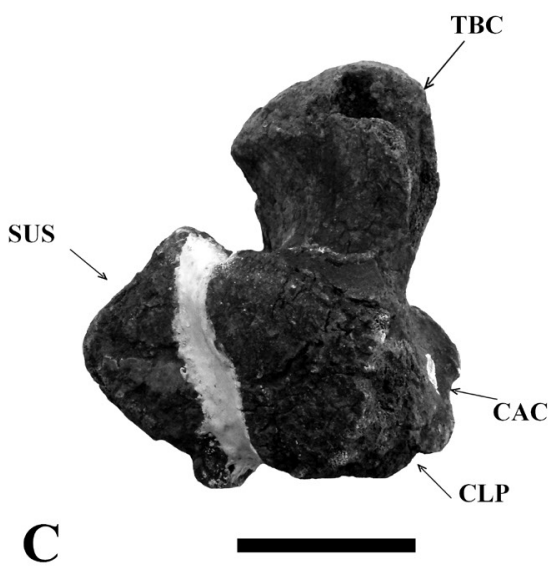

Figura 6 Proboscídeos fósiles hallados en la Barranca de San Mateo Huexoyucan, Tlaxcala, México. A, vista lateral de BUAPALZ-1031, cintura pélvica de Mammuthus sp. B, BUAPALZ-1026, calcáneo de Mammuthus sp. C, vista posterior de BUAPALZ-1062, calcáneo de Cuvieronius hyodon. La escala representa $15 \mathrm{~cm}$ en A, y $10 \mathrm{~cm}$ en B y C.

Tabla 5. Comparación de las medidas del ejemplar BUAPALZ-1026 y el calcáneo de Mammuthus reportado por Baygusheva et al., (2012). Medidas en milímetros ( $\mathrm{mm}$ ).

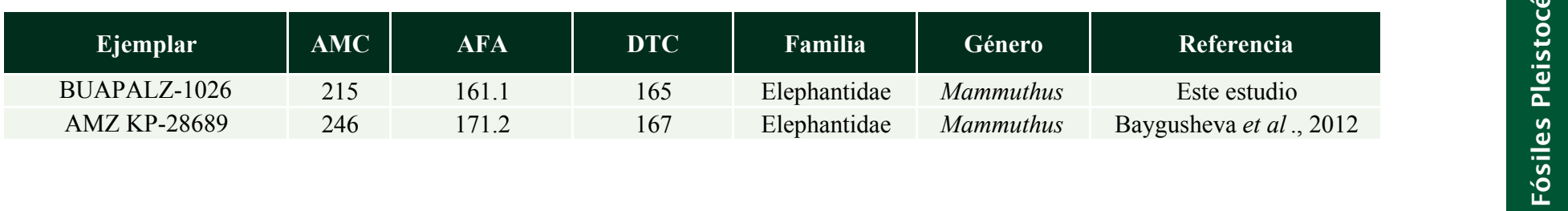


Tabla 6. Comparación del largo del ejemplar BUAPALZ-1062 y los ejemplares de calcáneo de Cuvieronius hyodon reportados por Tapia-Ramírez et al., 2013. Medidas en milímetros (mm).

\begin{tabular}{|c|c|c|}
\hline Ejemplar & (LC) & Referencia \\
\hline BUAPALZ -1062 & 169 & Este estudio \\
\hline DP 5811 & 169 & Tapia-Ramírez et al ., 2013 \\
\hline DP 5812 & 154 & Tapia-Ramírez et al ., 2013 \\
\hline DP 5813 & 166.2 & Tapia-Ramírez et al ., 2013 \\
\hline
\end{tabular}

A

B
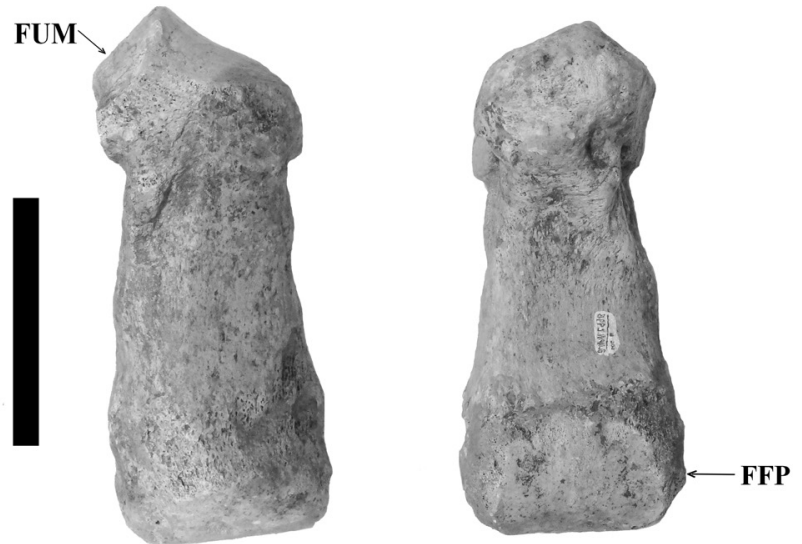

Figura 7 BUAPALZ-998, hueso del metacarpo IV vista palmar (A) dorsal (B) de Eremotherium laurillardi. Escala $=10 \mathrm{~cm}$

Material referido: BUAPALZ-1086, BUAPALZ-1087, BUAPALZ-1088, BUAPALZ-1089, osteodermos aislados (Tabla 7).

Descripción. El ejemplar BUAPALZ-1086 tiene contorno pentagonal, su figura central es redondeada, ligeramente cóncava y abarca la mitad del osteodermo, el surco marginal es visible, con dos folículos pilosos y siete figuras periféricas visibles pero fragmentadas y más pequeñas que la figura central (Figura 8 A). El ejemplar BUAPALZ-1087 está fragmentado, tiene contorno romboide, su figura central es redondeada, ligeramente cóncava, abarcando la mitad del osteodermo, el surco marginal es visible y a pesar de la fragmentación, se observan tres folículos pilosos y cuatro figuras periféricas pequeñas del lado derecho del osteodermo (Figura 8 B). El ejemplar BUAPALZ-1088 tiene un contorno pentagonal, su figura central es ligera-
A

B

C

D

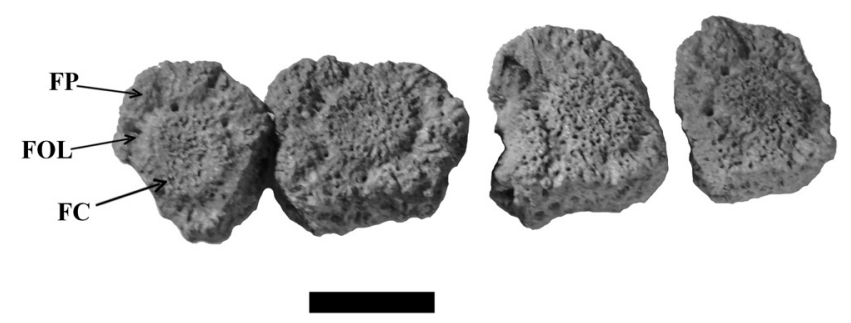

Figura 8 A, BUAPALZ-1086; B, BUAPALZ-1087; C, BUAPALZ1088; D, BUAPALZ-1089, osteodermos aislados de cf. Glyptotherium floridamun. Escala $=4 \mathrm{~cm}$.

mente cóncava y abarca la mitad del osteodermo, presenta el surco marginal con dos folículos pilosos y cinco figuras periféricas pequeñas (Figura $8 \mathrm{C}$ ). El ejemplar BUAPALZ-1089 es un osteodermo pentagonal, su figura central es circular y ligeramente cóncava, presenta un surco marginal con dos folículos pilosos y cuatro figuras periféricas incompletas más pequeñas que la figura central (Figura 8 D).

Discusión. Se han registrado cinco especies de Glyptotherium en América del Norte; tres de ellas para el Rancholabreano de México, las cuales son G. cylindricum, G. floridanum y G. mexicanum. Debido a la presencia del género Bison, la identificación se basó en las tres especies de gliptodontes rancholabreanas. $\mathrm{Al}$ ser comparados con los ejemplares de osteodermos de la región lateral referidos a Glyptotherium floridanum reportados por Bravo-Cuevas et al. (2009), los ejemplares BUAPALZ-1086, BUAPALZ-1087, BUAPALZ-1088 y BUAPALZ-1089, mostraron similitudes, ya que presentan la figura central ligeramente elevada y débilmente cóncava, como se aprecia en los osteodermos de Glyptotherium floridanum, en los cuales las figuras centrales son aproximadamente iguales que las periféricas, y por lo general son ligeramente levantadas y débilmente cóncavas (Gillette y Ray, 1981). Por su parte, el material de Tlaxcala difiere de las otras dos especies de gliptodontes, ya que G. cylindricum presenta figuras centrales con una superficie que varía de plana a ligeramente convexa y son más grandes que las periféricas; G. mexicanum se distingue por tener figuras centrales más grandes que las 
Tabla 7. Medidas de los osteodermos de Glyptotherium de la Barranca de San Mateo Huexoyucán, Tlaxcala, México. Medidas en milímetros $(\mathrm{mm})$.

\begin{tabular}{|c|c|c|c|c|c|}
\hline EJEMPLAR & DAPO & DAPFC & DTO & DTFP & $\begin{array}{c}\text { Número de figuras } \\
\text { periféricas visibles }\end{array}$ \\
\hline BUAPALZ-1086 & 40.3 & 19.99 & 32.97 & -- & 6 \\
\hline BUAPALZ-1087 & 41.43 & 21.13 & 18 & -- & 4 \\
\hline BUAPALZ-1088 & 42.24 & 20.68 & 34 & 9.98 & 8 \\
\hline BUAPALZ-1089 & 41.05 & 20.08 & 35 & -- & 5 \\
\hline
\end{tabular}

periféricas (Gillette y Ray, 1981; Mead et al., 2007), características ausentes en el material estudiado. Debido al número de ejemplares y a su estado de conservación, el material de San Mateo Huexoyucán se asignó tentativamente a G. floridanum.

Ramírez-Cruz y Montellano-Ballesteros (2014) proponen la sinonimia de G. floridanum con G. cylindricum con base en el estudio de dos esqueletos parciales; sin embargo, Gillette et al. (2015) consideran necesario evaluar más a fondo las relaciones entre G. cylindricum y las otras especies de gliptodontes para determinar su probable sinonimia.

\section{Discusión}

Los mamíferos identificados en la localidad 6 denominada "La Barranca de San Mateo Huexoyucán" son de hábitos alimentarios herbívoros: Bison antiquus, cf. Bison occidentalis, Camelops hesternus, cf. Glyptotherium floridanum y Eremotherium laurillardi, los cuales son nuevos registros para la localidad. Se reporta la presencia de proboscídeos como Mammuthus sp. y Cuvieronius hyodon, y équidos pleistocénicos como Equus conversidens, Equus sp., y Equus mexicanus, éste último también es nuevo registro para la localidad.

El registro de Bison antiquus permitió determinar la Edad Mamífero de Norteamérica del área de estudio como Rancholabreana y el fechado previo de la secuencia de paleosuelos permite ubicar a los fósiles aquí estudiados entre los 51798 - 47650 años A.P.

\section{Conclusiones}

En este trabajo se identificaron cinco órdenes (Artiodactyla, Cingulata, Perissodactyla, Proboscidea, Xenarthra), siete familias (Bovidae, Camelidae, Equidae, Elephantidae, Gomphotheriidae, Megatheriidae, Glyptodontidae), siete géneros (Bison, Camelops, Cuvieronius, Equus, Eremotherium, Glyptotherium y Mammuthus,) y nueve especies, de las cuales seis son nuevos registros para el área de estudio (Bison antiquus, cf. Bison occidentalis, Camelops hesternus, Eremotherium laurillardi, Equus mexicanus, cf. Glyptotherium floridanum). Tres especies reportadas anteriormente (Cuvieronius hyodon, Equus conversidens, Mamuthus sp.) se describen formalmente para la zona de San Mateo Huexoyucán. Estos ejemplares reflejan parte de la mastofauna pleistocénica de la Cuenca Puebla-Tlaxcala y el potencial paleontológico que ofrece esta localidad para futuros estudios paleontológicos.

\section{Agradecimientos}

Agradecemos a las autoridades y vecinos de la comunidad de San Mateo Huexoyucán, Tlaxcala por permitir la autorización para el trabajo de campo. A la Benemérita Universidad Autónoma de Puebla por el financiamiento para la investigación. A la M. en C. Laura Zayas Ocelotl por la ayuda en la revisión de la primera versión del manuscrito. A las biólogas Mariana Contreras López y Diana Karen Pérez Lara por su ayuda en la edición de fotografías, y a los revisores Alfredo E. Zurita y Nicolás Roberto Chimeto, por sus valiosas 
sugerencias y comentarios, los cuales ayudaron a mejorar este trabajo.

\section{Referencias}

Alonso-Aguilar, L.E., Montoya, A., Kong, A., Estrada-Torres, A., Garibay-Origel, R., 2014, The cultural significance of wild mushrooms in San Mateo Huexoyucán, Tlaxcala, México: Journal of Ethnobiology and Ethnomedicine, 10, 1-15.

Arroyo-Cabrales, J.,. Polaco O.J., Johnson, E., 2002, La mastofauna del Cuaternario tardío en México, en Montellano-Ballesteros, M., Arroyo-Cabrales, J. (coords.), Avances en los estudios paleomastozoológicos en México: México, D.F., Instituto Nacional de Antropología e Historia, Colección Científica, 103-123.

Arroyo-Cabrales, J., Carreño, A.L., LozanoGarcía, S., Montellano-Ballesteros, M., 2008, La Diversidad en el pasado, en Sarukhán, J., (Coord.), Soberón, J., Halffter, G., LlorenteBousquets, J., Capital natural de México, Conocimiento actual de la biodiversidad: México, CONABIO, 620 p.

Baskin, J., Thomas, R., 2016, A review of Camelops (Mammalia, Artiodactyla, Camelidae), a giant llama from the Middle and Late Pleistocene (Irvingtonian and Rancholabrean) of North America: Historical Biology, 28, 120-127.

Baygusheva, V.S., Titov, V.V., Timonina, G.I., 2012, Two skeletons of Mammuthus trogontherii from the Sea of Azov Region: Quaternary International, 276, 242-252.

Bell, C.J., Lundelius Jr., E.L., Barnosky, A.D., Graham, R.W., Lindsay, E.H., RuezJr., D.R., Semken Jr., H.A., Webb, S.D., Zakrzewsky, R.J., 2004, The Blancan, Irvingtonian and Rancholabrean Mammal Ages, en Woodburne, M.O. (ed.)., Late Cretaceous and Cenozoic mammals of North America: United States, Columbia University Press, 232-314.
Bonilla-Toscano, L., 2011, Descripción e identificación de la mastofauna fósil del municipio de Panotla, Tlaxcala: Puebla, México, Escuela de Biología BUAP, Tesis de Licenciatura, $52 \mathrm{p}$.

Bravo-Cuevas, V.M., Ortiz-Caballero, E., Cabral-Perdomo, M.A., 2009, Gliptodontes (Xenarthra, Glyptodontidea) del Pleistoceno Tardío (Rancholabreano) de Hidalgo, centro de México: Boletín de la Sociedad Geológica Mexicana, 61, 267-277.

Brookes, J., 1828, A catalogue of the anatomical and zoological museum of Joshua Brookes: London, Taylor, 1, 1-70.

Cabrera, A., 1929, Una revisión de los mastodontes argentinos: Revista del Museo de la Plata, $32,61-144$.

Cartelle, C., De Iuliis, G., 1995, Eremotherium laurillardi: The Panamerican Pleistocene Megatheriid Sloth: Journal of Vertebrate Paleontology, 15, 830-841.

Castañeda-Posadas, C., Calvillo-Canadell, L., Cevallos-Ferriz, S., 2009, Woods from Miocene sediments in Panotla, Tlaxcala, Mexico: Review of Paleobotany and Palynology, 159, 494-506.

Castillo-Cerón, J.M., Cabral-Perdomo, M.A., Carranza-Castañeda, O., 1996, Vertebrados fósiles del Estado de Hidalgo: México D.F., Universidad Autónoma de Hidalgo, 127 p.

Comisión Nacional para el Conocimiento y Uso de la Biodiversidad (CONABIO), 2012, Edad de Hielo (en línea), disponible en <http:// www.biodiversidad.gob.mx/biodiversidad/ EdHielo/edadHielo.html>, consultado 03 de octubre de 2016.

Cope, E.D., 1889, The Edentata of North America: American Naturalist, 23, 659-664.

Corona-M., E., Montellano-Ballesteros, M., Arroyo-Cabrales, J., 2005, Breve historia de la Paleomastozoología en México (resumen), en II Congreso Latinoamericano de Paleontología de Vertebrados: Río de Janeiro, Brasil, 82-84. 
De Iuliis, G., 1996, A Systematic review of the Megatheriinae (Mammalia: Xenarthra: Megatheriidae): Canada, Departement of Zoology University of Toronto, tesis doctoral, $805 \mathrm{p}$.

De la Treja-Segura, M.A., Sánchez-Garrido, E., Moctezuma-Salgado, M.D., De los SantosMontaño, J.J., 2014, Carta GeológicoMinera Ciudad de México E14-2, 1:250000 (en línea): Pachuca, Hidalgo, México, Servicio Geológico Mexicano, 1 mapa, disponible en <http://mapserver.sgm.gob. $\mathrm{mx} /$ cartas_impresas/productos/cartas/ cartas250/geologia/89_E14-2GM.html>, consultado 03 de octubre de 2016.

Fischer, G., 1814, Zoognosia. Tabulis synopticis illustrate: Moscow, Typis Nicolai Sergeidis Vsevolozsky, 3, 1-694.

Gillette, D.D., Ray, G.E. 1981, Glyptodonts of North America: Smithsonian Contributions to Paleobiolology, 40, 1-255.

Gillette, D., Carranza-Castañeda, O., White, R.S.Jr., Morgan, G., Thraser, L.C., McCord, R., McCullough, G., 2015, Ontogeny and Sexual Dimorphism of Glyptotherium texanum (Xenarthra, Cingulata) from the Pliocene and Pleistocene (Blancan and Irvingtonian NALMA) of Arizona, New Mexico, and Mexico: Journal of Mammalian Evolution, $22,1-22$.

Gray, J.E., 1821, On the natural arrangement of vertebrose animals: London Medical Repository Review, 15, 296-310.

Gray, J.E., 1869, Catalogue of carnivorous, pachydermatous, and dentate mammalia in the British Museum: London, British Museum Natural History, 398 p.

Guenther, E.W., Bunde, H., 1973, Investigaciones geológicas y paleontológicas en México durante los años de 1965 a 1969: Comunicaciones, 7, 19-20.

Hibbard, W.C., 1955, Pleistocene vertebrates from the Upper Becerra (Becerra Superior)
Formation, Valley of Tequixquiac, México, with notes on other Pleistocene forms: Contributions from the Museum of Paleontology, University of Michigan, 21, 47-96.

Illiger, C., 1811, Prodromus systematis mammalium et avium additis terminis zoographicis utriusque classis, eorumque versione germanica (en línea): Berlin, Salfeld, 1, 1-301, disponible en <http://dx.doi. org/10.5962/bhl.title.106965>, consultado 03 de octubre de 2016.

Jiménez-Hidalgo, E., Cabrera-Pérez, L., MacFadden, B.J., Guerrero-Arenas, R., 2013, First record of Bison antiquus from the late Pleistocene of southern México: Journal of South American Earth Sciences, 42, 83-90.

Leidy, J., 1852, Memoir on the extinct species of American ox: Smithsonian Contributions. Knowledge, 5 (3), 1-20.

Leidy, J., 1854, On Bison latifrons, Arctodus pristinus, Hippodon speciosus, and Merycodus sabulonis: Proceedings of the Academy of Natural Sciences of Philadelphia, 7, 89-90.

Leidy, J., 1873, Contributions to the extinct vertebrate fauna of the western Territories: United States geological and geographical survey of the Territories, 1, 14-358.

Lermo-Samaniego, J., Bernal-Esquia, I., 2006, Zonificación sísmica del estado de Tlaxcala, México: Boletín de la Sociedad Geológica Mexicana, 58, 215-221.

Linnaeus, K., 1758, Systema naturae perregnatria naturae, secundum classes, ordines, genera, species cum characteribus, differentiis, synonymis, locis: Stockholm, Laurentii Salvii Editiodecima, reformata, $824 \mathrm{p}$.

Lucas, S.G., Alvarado G.E., 2010, Fossil Proboscidea from the Upper Cenozoic of Central America: Taxonomy, evolutionary and paleobiogeographic significance: Revista Geológica de América Central, 42, 9-42. 
Lund, P.W., 1842, Blik paa Brasiliens Dyreverden försidstejordomvaeltning. Fjerde Afhandling: Fortsaettelse af Pattedyrene. Lagoa Santa d. 30 Januar 1841. Copenhague. -K. Danske videnskabernes Selskabs naturvidenskapelige og mathematiske Afhandlinger, 9, 137-208.

Martinelli, A.G., Fonseca-Ferraz, P., CardosoGunha, G., 2012, First record of Eremotherium laurillardi (Lund, 1842) (Mammalia, Xenarthra, Megatheriidae) in the Quaternary of Uberaba, TriânguloMineiro (Minas Gerais State), Brazil: Journal of South American Earth Sciences, 37, 202-207.

McDonald, J.N., 1981, North American Bison: Their classification and evolution: California, USA, University of California Press, Berkeley, California, 1-316.

Mead, J.I., Swift, S.L., White, R.S., McDonald, H.G, Báez, A., 2007, Late Pleistocene (Rancholabrean) Glyptodont and Pampathere (Xenarthra, Cingulata) from Sonora, México: Revista Mexicana de Ciencias Geológicas, 24, 439-449.

Metallo, A., Blundell, J., Rossi V., 2010, Smithsonian X 3D Beta Woolly Mammoth Model (en línea): Washington, DC, Digitization Program Office Smithsonian Institution, disponible en <http://3d.si.edu/ explorer?modelid $=55>$, consultado 03 de octubre de 2016

Osborn, H.F., 1903, Glyptotherium texanum, a new glyptodont, from the Lower Pleistocene of Texas: Bulletin of the American Museum of Natural History, 19, 491-494.

Osborn, H.F., 1921, The evolution, phylogeny, and classification of the Proboscidea: American Museum Novitates, 1, 1-14.

Osborn, H.F., 1923, New subfamily, generic, and specific stages in the evolution of the Proboscidea: American Museum Novitates, 99, 1-4.

Oslen, J.S., 1960, Post-cranial skeletal characters of Bison and Bos: Cambridge, Massachusetts,
Papers of the Peabody Museum of Archaeology and Ethnology Harvard University, $15 \mathrm{p}$.

Owen, R., 1848, Description of the teeth and portions of jaws of two extinct anthracotheroid quadrupeds discovered by the Marchioness of Hastings in the Eocene deposits of the N.W. coast of the Isle of Wight: with an attempt to develop Cuvier's idea of the classification of Pachyderms by the number of their toes: Quarterly Journal Geological Society of London, 4, 103-141.

Owen, R., 1869, On Fossil Remains of Equines from Central and South-America referable to Equus conversidens $\mathrm{Ow}$., and $E$. arcidens $\mathrm{Ow}$ : Philosophical Transactions of the Royal Society of London, 159, 559-73.

Ramírez-Cruz, G.A., Montellano-Ballesteros, M., 2014, Two news Glyptodonts records (Mammalia: Cingulata) from the Late Pleistocene of Tamaulipas and Tlaxcala, Mexico: Implications for the taxonomy of the genus Glyptotherium: The Southwestern Naturralist, 59, 522-530.

Raymond, K.R., Prothero, D.R., 2011, Did climate changes affect size in Late Pleistocene Bison?: New Mexico Museum of Natural History and Science Bulletin, 53, 636-640.

Schmid, E., 1972, Atlas of animal bones: for prehistorians and Quaternary geologist: Amsterdam, The Netherlands, Elsevier, 153 p.

Sedov, S., Solleiro-Rebolledo, E., Terhorst, B., Solé, J., Flores-Delgadillo, M.L., Werner, G., Poetsch, T., 2009, The Tlaxcala basin paleosol sequence: A multiscale proxy of middle to late Quaternary environmental change in central México: Revista Mexicana de Ciencias Geológicas, 26, 448-465.

Sisson, J.D., Grossman R.G., 1978, Anatomía de los animales domésticos: Madrid, España. Salvat Editores quinta edición, tomo 1, 1335 p. 
Solís-Castillo, B., Solleiro-Rebolledo E., SalcidoBerckovich C., 2012, Paleosuelos en secuencia coluvo-aluviales del PleistocenoHoloceno en Tlaxcala: registros paleo ambientales del poblamiento temprano en el centro de México: Boletín de Sociedad Geológica Mexicana, 64, 91-108.

Smith, C.H., 1827, Supplement to the order Ruminantia, en Cuvier, G., The animal kingdom arranged in conformity with its organization: London, United Kingdom, Geo. B. Whittaker, 4, 373.

Spillmann, F., 1948, Beitrge zur Kenntnis eines neuen gravigraden Riesensteppentieres (Eremotherium carolinenese gen. et. spec. nov.), seines Lebensraumes und seiner Lebensweise: Palaeobiologica 8, 3, 231-279.
Tapia-Ramírez, G., Guzmán, A.F., Polaco, J.O., 2013, Los Gonfoterios (Proboscidea, Gomphoteriidae) de Colima, México: Boletín de la Sociedad Geológica Mexicana, 65, 591-607.

Tovar, R.E., Sedov, S., Solís, B., Solleiro, E., 2013, Dark humic alluvial paleosols in central and southern Mexico: micromorphological indicators of late Pleistocene megafauna habitats: Spanish Journal of Soil Science 3, 217-235.

Vilaclara, G., Martinez-Mekler, G., Cuna, E., Ugalde, E., 2009, Diatom-inferred palaeoenvironmental changes of a Pliocene lake disturbed by volcanic activity: Journal of Paleolimnology, 44, 203-215.

\section{Apéndice}

\section{Abreviaturas anatómicas}

Acetábulo

Acromion

Alveolos

Apófisis palatina

Canal posterior

Cara articular para el astrágalo

Cara astrágalo y cuboides

Cara lunar para el astrágalo

Cuello de la escápula

Diáfisis proximal

Diente canino

Espina escapular

Faceta articular del húmero

Faceta ectomesucneiforme

Faceta hueso cuneiforme

Faceta hueso ectocuneiforme

Faceta para el unciforme y metacarpo

Faceta para la falange proximal

Figura central

Figura periférica

Folículo piloso
$\mathrm{ACB}$

ACR

AVS

APT

CP

CAA

CAC

CLP

CE

DP

$\mathrm{CN}$

ESE

FAH

FEM

FHC

FHE

FUM

FPP

FC

FP

FOL

\section{Abreviaturas anatómicas}

\section{Foramen}

Foramen obturador

Fosa oval

Hendidura acetabular

Ilion

Isquion

Labio del acetábulo

Mesostilo

Metastilo

Molar

Muesca acetabular

Parastilo

Plihipostilo

Pliprotolofo

Postfoseta

Prefoseta

Proceso coracoideo

Protocono

Pubis

Sustentáculo

Tuberosidad calcánea 


\section{Abreviaturas de medidas}

Altura máxima del calcáneo

Ancho de la epífisis

Ancho de la faceta para el astrágalo

Ancho del tarso

Diámetro anteroposterior del osteodermos

Diámetro anteroposterior de la figura central

Diámetro del acetábulo

\section{Abreviaturas de medidas}

$\mathrm{AMC}$

$\mathrm{AE}$

AFA

AT

DAPO

DAPFC

DA
Diámetro transversal de una de las figuras periféricas Diámetro transversal del osteodermo

DTFP

Diámetro transversal del tubérculo del calcáneo

DTO

DTC

LA

Largo del acetábulo

LC

LTC 